\title{
Análisis de la Revista Biomédica en sus diez primeros años.
}

\section{Artículo Original}

Fernando J. Álvarez-Cervera, José L. Góngora-Alfaro, José L. Bata-García, Francisco J. Heredia-López.

Laboratorio de Neurofisiología. Centro de Investigaciones Regionales “Dr. Hideyo Noguchi”, Universidad Autónoma de Yucatán, Mérida, Yucatán, México.

\section{RESUMEN.}

Introducción. La Revista Biomédica celebró su décimo aniversario en 1999 con la publicación del número 4 del volumen 10. En este trabajo se presenta un análisis del contenido de la revista hasta ese número, así como un resumen de las opiniones de una muestra de sus lectores.

Material y Métodos. Las opiniones de los lectores y los bibliotecarios fueron recabadas por medio de una encuesta que se les envió por correo. También se analizó el contenido de la revista, contabilizando el número de artículos de acuerdo a su tipo, a su contenido y al lugar de adscripción de los autores. Se estudió también la composición del Comité Editorial, así como la participación de árbitros. Asimismo, se investigaron otros elementos tales como la referencia hecha en publicaciones externas a los artículos publicados en la propia revista, y el porcentaje de contribuciones rechazadas.

Resultados. Se recibieron 142 cuestionarios contestados por lectores $(15.3 \%$ del total que se enviaron), y 7 de bibliotecarios (6.4\% de los 109 que se distribuyeron). Las respuestas se refieren al lugar de adscripción de los lectores, al tipo de actividad preponderante que desarrollan, al tipo y cantidad de artículos que leen, al hecho de citar trabajos de la revista en otras publicaciones y a la opinión general que tienen de la revista, entre otros puntos.

Discusión. Con base en estos resultados y en el análisis de la evolución del contenido de la revista se proponen algunos cambios que pudieran contribuir a su progreso. (Rev Biomed 2000; 11:235-249)

Palabras clave: Revista Biomédica, revistas médicas.

\section{SUMMARY.}

Analysis of the Revista Biomédica in its first ten years.

Introduction. The Revista Biomédica celebrated its tenth anniversary with the publication of number 4 in volume 10 . In this work an analysis of the contents of the journal up to that issue is presented, as well as a summary of the opinions of a sample of its readers.

Materials and Methods. The opinions of the

Solicitud de sobretiros: M. en C. Fernando J. Álvarez-Cervera. Laboratorio de Neurofisiología. Centro de Investigaciones Regionales "Dr. Hideyo Noguchi”, Universidad Autónoma de Yucatán. Av. Itzáes No. 490 x 59. Tel. (99)24-64-12; Fax: (99) 23-61-20. E-mail: acervera@tunku.uady.mx Recibido el 2/Octubre/2000. Aceptado para publicación el 3/Noviembre/2000. 


\section{FJ Álvarez-Cervera, JL Góngora-Alfaro, JL Bata-García, FJ Heredia-López.}

readers were gathered by means of a survey sent to them by mail. One questionnaire was used for readers, and another one for librarians. The contents of the journal were analyzed, counting the number of articles according to type, subject, and place of affiliation of the authors. The composition of the Editorial Committee was also studied, as well as the participation of referees. Likewise, other elements, such as references made in external publications of papers that appeared in the journal, and the percentage of rejected contributions, were investigated.

Results. 142 completed questionnaires from readers (15.3\% of the total sent), and $7(6.4 \%)$ out of 109 from librarians, were received. The answers refer to the place of affiliation of the readers, to the primary activity they perform, to the type and number of articles they read, to the references in other publications to papers published in this journal, and to the general opinion they have of the journal, among other issues.

Discussion. Based on these results and on the analysis of the evolution in the contents of the journal, some changes which could contribute to its progress are proposed. (Rev Biomed 2000; 11:235-249)

Key words: Revista Biomédica, medical journals.

\section{INTRODUCCIÓN.}

La Revista Biomédica fue creada por el Centro de Investigaciones Regionales "Dr. Hideyo Noguchi" (CIR) y la Facultad de Medicina de la Universidad Autónoma de Yucatán (UADY). En el artículo editorial correspondiente al primer número publicado a principios de 1990, el entonces director del CIR, Dr. Jorge Zavala-Velázquez, afirmaba que la revista pretendía en su primera etapa constituir un medio de unión entre la comunidad médica de la región, pero con la aspiración de trascender gradualmente a una zona más amplia (1). Cinco años más tarde, el Dr. Renán A. Góngora-Biachi, en su carácter de editor de la revista, escribía en la columna editorial que la
Revista Biomédica ya contenía contribuciones de autores de diversos Estados de la República Mexicana y del extranjero, e igualmente incluía un número importante de suscriptores foráneos. Reconocía también que la publicación, como otras revistas biomédicas mexicanas, enfrentaba dos retos importantes. El primero era el de lograr que fuera considerada equiparable a las publicaciones científicas internacionales. El segundo era vencer el obstáculo de valorar una revista únicamente con base en su factor de impacto, obtenido de un índice internacional líder de citas científicas (2). Uno de estos índices, el Science Citation Index (SCI), engloba a cerca de 3,300 revistas consideradas como las de más alto nivel, de entre aproximadamente 70,000 que se editan a nivel mundial, según cifras de 1994 (3). De las revistas comprendidas en el índice, sólo 50 (el 1.5\%) eran editadas en países en desarrollo. Y dentro del SCI, sólo el $0.33 \%$ de los trabajos (1 de cada 300) era de investigadores mexicanos.

Estas preocupaciones eran también compartidas por la editora de la Revista Española de Paleontología (4). Ella consideraba que el uso de estos índices de publicaciones científicas como un medio de evaluación de la calidad de los artículos no es siempre procedente. Con respecto a lo inadecuado de utilizar el SCI para calcular el factor de impacto de una publicación, argumentaba que éste está basado únicamente en las citas que aparecen en las revistas ya incluidas en el propio índice, lo cual puede no coincidir con el número real de referencias. Más aún, hacía notar que no se toma en cuenta el hecho de que los distintos campos de la ciencia son de interés para un número dispar de investigadores y, entonces, la comparación de los impactos de trabajos que tratan de disciplinas diferentes no es una medida fiel de su calidad relativa. Por otra parte, el hecho de que el número de revistas mexicanas comprendidas en el SCI sea bajo o nulo constituye una razón para que los autores manden sus originales a revistas extranjeras que sí estén incluidas en el índice. Esto trae consecuencias como la disminución en la cantidad de manuscritos que se someten a las revistas mexicanas, y el desinterés de los investigadores por

\section{Revista Biomédica}




\section{Análisis de la Revista Biomédica.}

temas científicos de importancia local o regional.

Al cabo de diez años, las tendencias de crecimiento e internacionalización de la Revista Biomédica seguían aumentando, según se describía en el artículo editorial correspondiente (5). Uno de los logros que se reportaban era el de haber conseguido la inclusión de la Revista Biomédica en varios índices y compendios mexicanos: en el llamado BIBLIO-MEX SALUD y en los discos compactos ARTEMISA 4, 5, 6 y 7, todos del Centro Nacional de Información y Documentación sobre Salud (CENIDS) de la Secretaría de Salud de México; en el Anuario Bibliográfico de Investigación en Salud del Instituto Mexicano del Seguro Social; y en el Índice Mexicano de Revistas Biomédicas (IMBIOMED), editado por la empresa Medinet, SA de CV. Asimismo, para 1999 la Revista Biomédica ya aparecía en cuatro índices internacionales: PERIÓDICA, Índice de Revistas Latinoamericanas en Ciencias, editado por el Centro de Información Científica y Humanística de la Universidad Nacional Autónoma de México; en el Sistema Latinoamericano y del Caribe de Información en Ciencias de la Salud (LILACS) editado por la Organización Panamericana de la Salud; en la colección Latinoamericana Benson, de la Universidad de Texas, EUA; y en el Índice de Periódicos y Revistas Españolas e Hispanoamericanas editado en Barcelona, España. Cabe señalar que la inclusión de la Revista Biomédica en el compendio ARTEMISA significa que ésta se situó dentro de las mejores (alrededor de 30) revistas biomédicas publicadas en México (6). Sin embargo, el Dr. Góngora Biachi es una de las personas que consideran que todavía persiste la impresión de que a los artículos publicados en las revistas biomédicas latinoamericanas, se les asigna un escaso valor para los sistemas de evaluación académica de las universidades y de otras entidades mexicanas, con respecto a las publicaciones en revistas incluidas en índices internacionales de primer orden .

Desde su creación, la Revista Biomédica ha experimentado varios cambios, la mayoría de ellos positivos. No obstante, consideramos pertinente efectuar un análisis detallado de la evolución de la revista, el cual permita apreciar los logros que se han alcanzado con ella como medio de comunicación y reflexión en el ámbito biomédico. Además, el análisis presentado en este trabajo puede conducir a proponer mejoras adicionales en cuanto al contenido, la calidad y la difusión de esta publicación científica.

\section{MATERIAL Y MÉTODOS.}

La información presentada está basada, en parte, en la inspección de los 40 números contenidos en los primeros diez volúmenes de la Revista Biomédica, que comprenden el período de 1990 a 1999. Por otro lado, se obtuvieron las opiniones de alrededor de 150 lectores y bibliotecarios que reciben periódicamente la revista.

\section{Encuesta.}

Se clasificó a los suscriptores de la Revista Biomédica en las categorías de usuarios personales y responsables de bibliotecas. Con el objeto de conocer las características y opiniones de los lectores personales se elaboró un cuestionario de 11 puntos (Anexo) que fue enviado junto con el número 2 del volumen 10. A los bibliotecarios se les envió un cuestionario de 4 puntos:

1.- Averiguar si en la biblioteca de adscripción se contaba o no con la colección completa (Vols. 1 al 9) de la Revista Biomédica. El volumen 10 no se incluyó debido a que aún no se habían distribuido los 2 últimos números.

2.- Conocer un estimado del número de consultas hechas a la Revista Biomédica durante el periodo 1996-1998.

3.- Indagar si las bibliotecas estaban en posibilidad de pagar por la Revista Biomédica, o bien, de ofrecer otra revista a cambio.

4.- Conocer sus comentarios y/o sugerencias.

Ambos cuestionarios fueron traducidos al inglés para los suscriptores de países no hispanoamericanos. 


\section{FJ Álvarez-Cervera, JL Góngora-Alfaro, JL Bata-García, FJ Heredia-López.}

Para los suscriptores personales se repartieron 922 cuestionarios en español y 7 en inglés. Para los responsables de bibliotecas se distribuyeron 102 en español y 7 en inglés. Además de las respuestas a los reactivos de las encuestas, se consideró como otra variable el lugar de adscripción de los lectores o bibliotecarios.

\section{Clasificación de los artículos publicados.}

Sólo se analizaron los tres tipos de artículos más leídos por los suscriptores personales que contestaron la encuesta: artículos originales, de revisión y casos clínicos. Se contabilizaron los artículos de estas clases publicados en cada uno de los diez primeros volúmenes de la Revista Biomédica (4 números por volumen), y los resultados se expresaron como fracciones de la suma total de artículos publicados en las tres categorías. Además, se consideraron cuatro categorías según la adscripción del autor principal (del CIR, de la Facultad de Medicina Veterinaria y Zootecnia de la UADY, de otras facultades de la UADY, y de instituciones externas). En este análisis no se incluyeron artículos que aparecen como parte de congresos o simposios.

Una posible norma para evaluar la calidad de una revista es el porcentaje de artículos publicados por autores externos, es decir, que no pertenecen a la institución que edita la revista. Por consiguiente, se hizo un análisis de la procedencia de los autores que publicaron artículos originales o de revisión en cada volumen de la Revista Biomédica. Los resultados se expresaron en porcentaje de artículos publicados por autores de alguna de las siguientes procedencias (y que representan las fuentes más numerosas de contribuciones a la revista): 1) Centro de Investigaciones Regionales (CIR) "Dr. Hideyo Noguchi", 2) Facultad de Medicina Veterinaria y Zootecnia de la Universidad Autónoma de Yucatán (UADY), 3) otras facultades de la UADY y 4) otras instituciones mexicanas o extranjeras.
Análisis de la composición del Comité Editorial. Otro de los criterios que se puede usar para evaluar la calidad de una revista es la diversidad de origen de los miembros del Comité Editorial. Para ello, se contabilizó el número de miembros del Comité Editorial en cada volumen, y se calculó el porcentaje de miembros de acuerdo con cada una de las siguientes procedencias: 1) personal adscrito al CIR "Dr. Hideyo Noguchi", 2) de otras facultades de la UADY, 3) de otras instituciones de la República Mexicana y 4) de instituciones extranjeras.

Análisis de la participación interna de árbitros.

Con base en las notas de agradecimiento a revisores, incluidas en ciertos números de la revista, se pudo hacer una estimación de la cantidad de evaluadores internos (adscritos al CIR) y externos que colaboran en el arbitraje de artículos de la Revista Biomédica.

\section{Obtención de datos complementarios.}

Dado que la Revista Biomédica actualmente no aparece en bases de datos bibliográficas mundiales del área médica, tales como Medline, no fue posible hacer un estudio de la evolución del impacto que ha tenido la revista. Sin embargo, con datos de las encuestas y de las carpetas de reimpresos de autores internos que se archivan en la biblioteca del CIR se pudo hacer un somero recuento de las citas a los trabajos publicados en la Revista Biomédica que han aparecido en revistas mexicanas o internacionales con arbitraje.

Por otra parte, se obtuvo información del Editor acerca de los porcentajes de aceptación (o de rechazo) de los trabajos sometidos a la Revista Biomédica y a otras revistas mexicanas.

\section{RESULTADOS.}

Encuesta.

Las respuestas se empezaron a recibir desde una semana después de que comenzó la distribución, hasta 11 meses después. De un total

\section{Revista Biomédica}


Análisis de la Revista Biomédica.

de 1038 cuestionarios enviados se recibieron 149 (14.4\%). De los 929 cuestionarios enviados a lectores personales, se recibieron 142 respuestas (15.3\%), mientras que de los 109 cuestionarios para bibliotecarios, sólo contestaron 7 (6.4\%). Esto sugiere que las respuestas son más representativas de las opiniones de los suscriptores personales.

De los 149 cuestionarios recibidos, 73 (49.0\%) fueron de personas que laboran en la UADY y 76 (51.0\%) de personas adscritas a otras instituciones mexicanas o del extranjero. Sin embargo, al analizar el número de respuestas dentro de cada categoría, se observó que el cuestionario fue respondido por el $42.9 \%$ de $\operatorname{los} 170$ encuestados de la UADY, en contraste con el $8.8 \%$ de los 868 encuestados externos. Por tal motivo se hizo una prueba de $\chi^{2}$ de dos colas con corrección de Yates para determinar la existencia de algún sesgo en la proporción de encuestas respondidas por ambos grupos:

\begin{tabular}{ccc}
\hline RESPONDIERON & UADY & EXTERNOS \\
\hline SI & 73 & 76 \\
NO & 97 & 792 \\
\hline
\end{tabular}

$$
\begin{aligned}
& \chi^{2}=132.4 \\
& \text { grados de libertad: } 1 \\
& \mathrm{P}<0.00001 \\
& \text { Razón de momios (“odds ratio") }=7.84
\end{aligned}
$$

Estos resultados son muy claros e indican que los encuestados de la UADY respondieron 7.84 veces más que los suscriptores externos, incluyendo a los bibliotecarios. Esto debe tomarse en consideración al interpretar las gráficas siguientes, donde se analizan de manera global las respuestas de suscriptores internos y externos.

En la figura 1A se ilustran las cantidades (y porcentajes) de suscriptores que respondieron a la encuesta, de acuerdo con una clasificación según su sitio de trabajo. Se incluye tanto a usuarios personales como a bibliotecarios. En la figura 1B aparece la distribución de los lectores personales de acuerdo con su actividad profesional. En esta pregunta muchos lectores marcaron más de una categoría, por lo que sus respuestas fueron contabilizadas individualmente. Otra aclaración importante que aplica tanto para esta figura como para las siguientes es que un pequeño número de lectores no contestaron algunas de las preguntas del cuestionario. Por lo tanto, el $100 \%$ en cada caso corresponde al total de encuestas que tuvieron respuesta para la pregunta en cuestión. La figura 1C es una representación de la cantidad de artículos de la Revista Biomédica que los suscriptores personales leen, en promedio, por número. En la figura 1D se presentan los tres tipos de artículos que los lectores acostumbran examinar con más frecuencia; en general, los encuestados que respondieron marcaron más de un tipo de artículo. La figura $1 \mathrm{E}$ se refiere al número de personas que leen el ejemplar de cada lector. En la figura $1 \mathrm{~F}$ está la representación gráfica de las respuestas que dieron los lectores a la pregunta sobre la cantidad de números o artículos que conservan de la Revista Biomédica.

En la figura $2 \mathrm{~A}$ se puede observar la distribución de los lectores con respecto a su participación como autores de artículos publicados en la revista, o su disposición a serlo. La figura 2B ilustra la cantidad de autores encuestados que ha hecho referencia a sus artículos publicados en la Revista Biomédica en otras publicaciones científicas. La figura $2 \mathrm{C}$ refleja las opiniones de los suscriptores acerca de la calidad de la revista en comparación con otras revistas biomédicas que acostumbran consultar. La figura 2D representa la opinión de los encuestados sobre la pregunta de si la Revista Biomédica debería publicarse únicamente en formato electrónico o no. La figura $2 \mathrm{E}$ se refiere al costo que lectores y bibliotecarios estarían dispuestos a pagar por la suscripción anual a la Revista Biomédica.

El último reactivo de la encuesta solicitaba las sugerencias o comentarios de los encuestados. Sesenta y cuatro lectores o bibliotecarios $(43.5 \%$ del total que respondieron a la encuesta) hicieron alguna anotación en este rubro. Sus respuestas pueden clasificarse de manera general en cuatro áreas principales: temática de la revista (20

Vol. 11/No. 4/Octubre-Diciembre, 2000 
FJ Álvarez-Cervera, JL Góngora-Alfaro, JL Bata-García, FJ Heredia-López.

ADSCRIPCIÓN DE LOS LECTORES

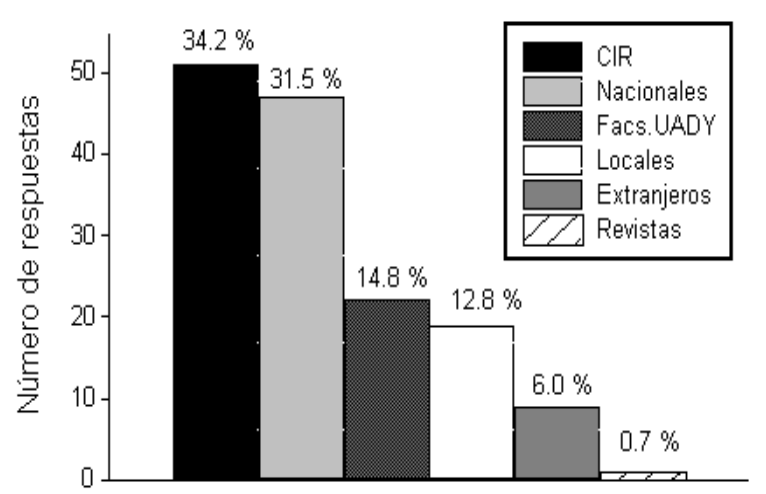

A

FRECUENCIA DE LECTURA

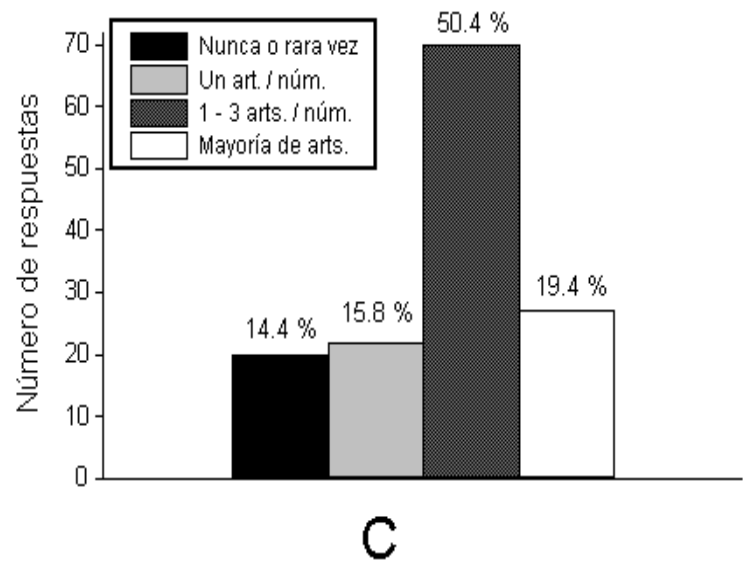

LECTORES POR EJEMPLAR

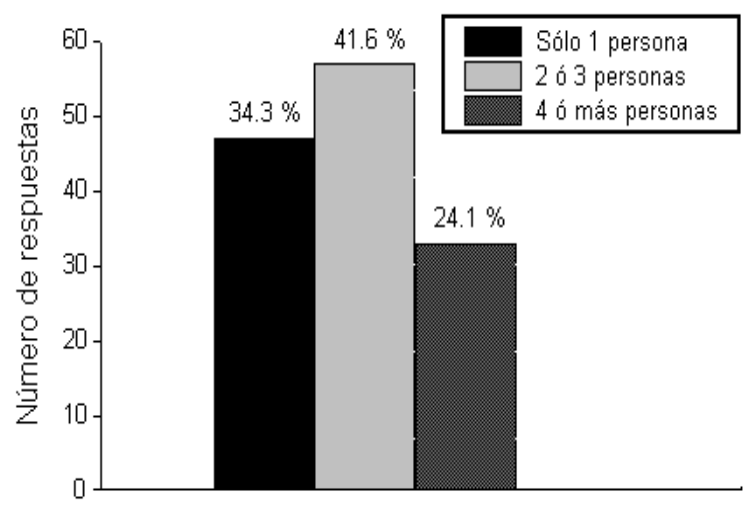

E
ACTIVIDADES DE LOS ENCUESTADOS

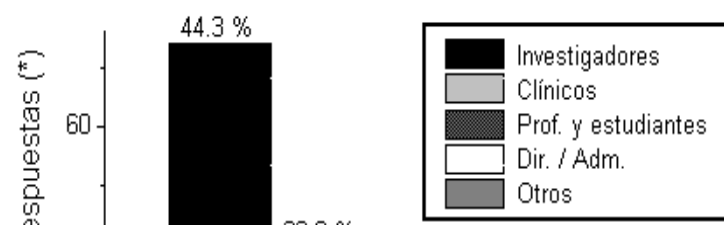

$22.8 \%$

$16.8 \%$

$8.4 \% \quad 7.7 \%$

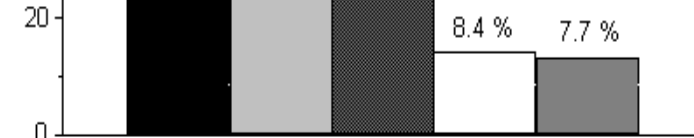

B

3 TIPOS DE ARTÍCULOS MÁS LEÍDOS

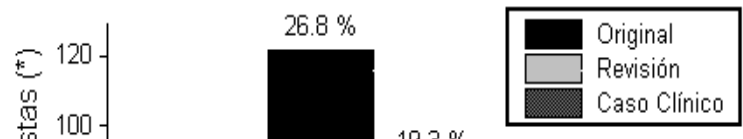

$19.3 \%$

$13.4 \%$

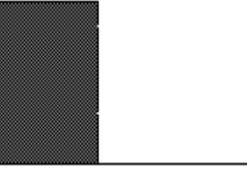

NÚMEROS QUE CONSERVA

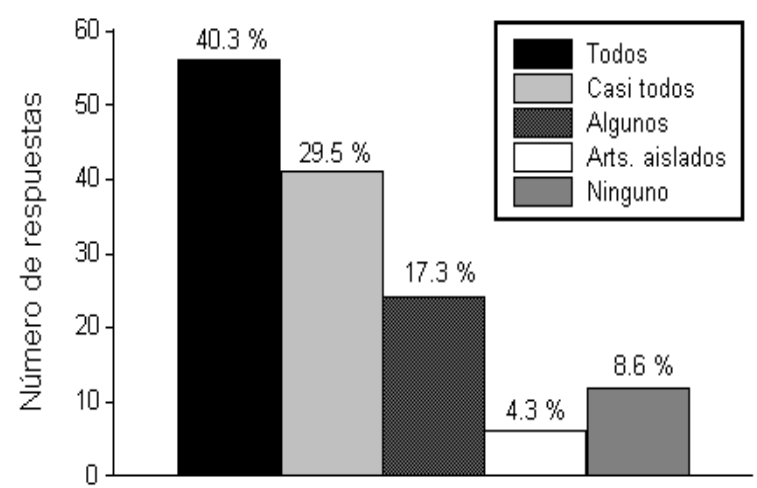

F

Figura 1.- Representación gráfica de las respuestas de los lectores de la Revista Biomédica a las preguntas de la encuesta ( $1^{\mathrm{a}}$ parte). Los números sobre las barras indican el porcentaje de respuestas para cada categoría, con respecto al total de respuestas recibidas para la pregunta en cuestión. Las alturas de las barras corresponden al número de lectores que dieron cada categoría de respuesta.

* Para estas preguntas algunos lectores dieron más de una respuesta 
Análisis de la Revista Biomédica.

AUTORÍA DE LOS LECTORES

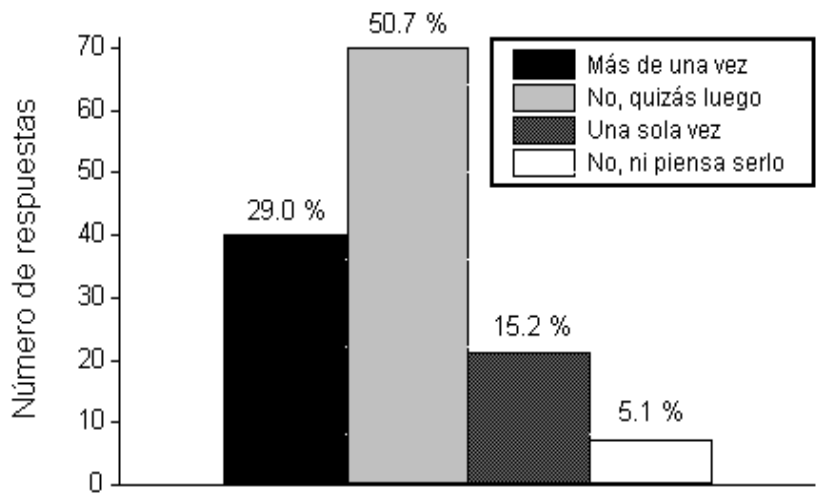

A

CALIDAD DE LA REVISTA

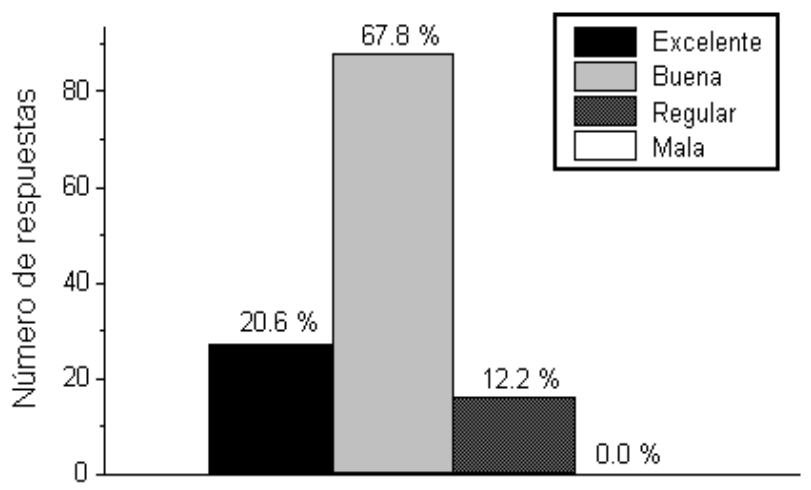

C

POSIBLE PAGO POR LA REVISTA

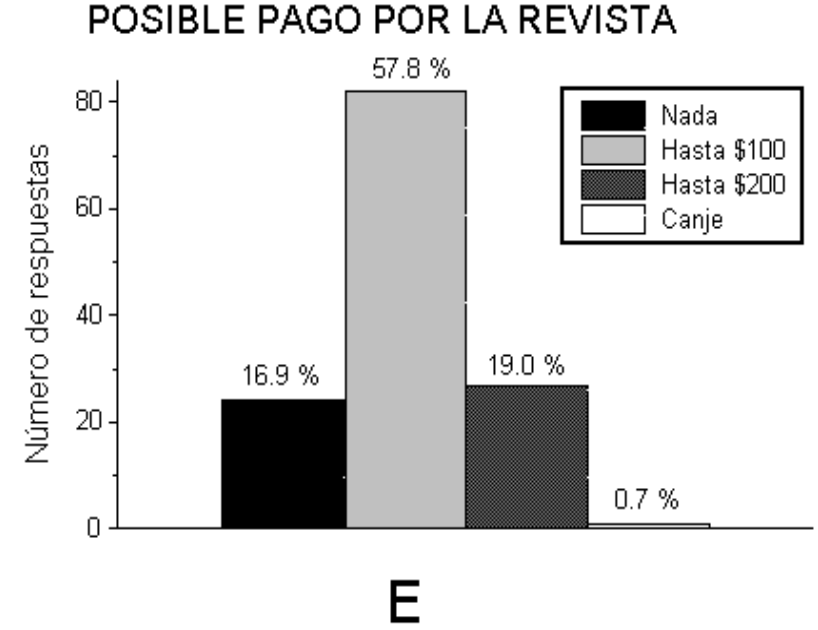

E
REFERENCIAAARTÍCULOS

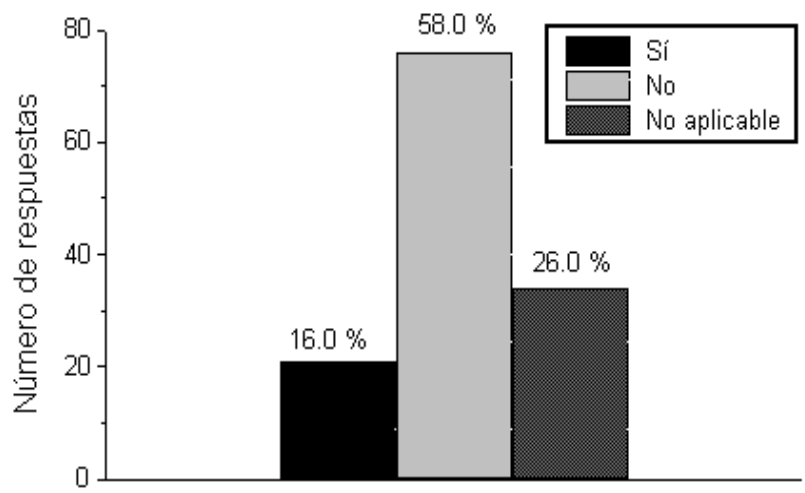

B
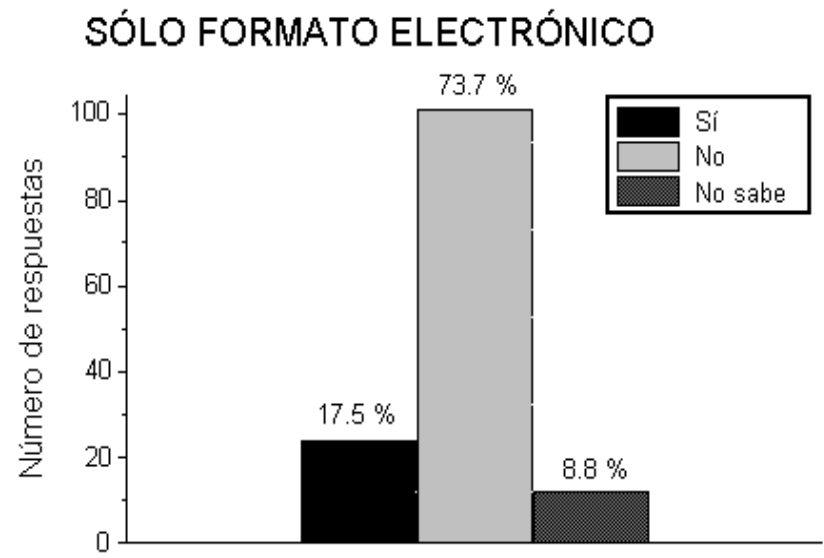

D

Figura 2.- Representación gráfica de las respuestas de los lectores de la Revista Biomédica a las preguntas de la encuesta ( $2^{\mathrm{a}}$ parte). 


\section{FJ Álvarez-Cervera, JL Góngora-Alfaro, JL Bata-García, FJ Heredia-López.}
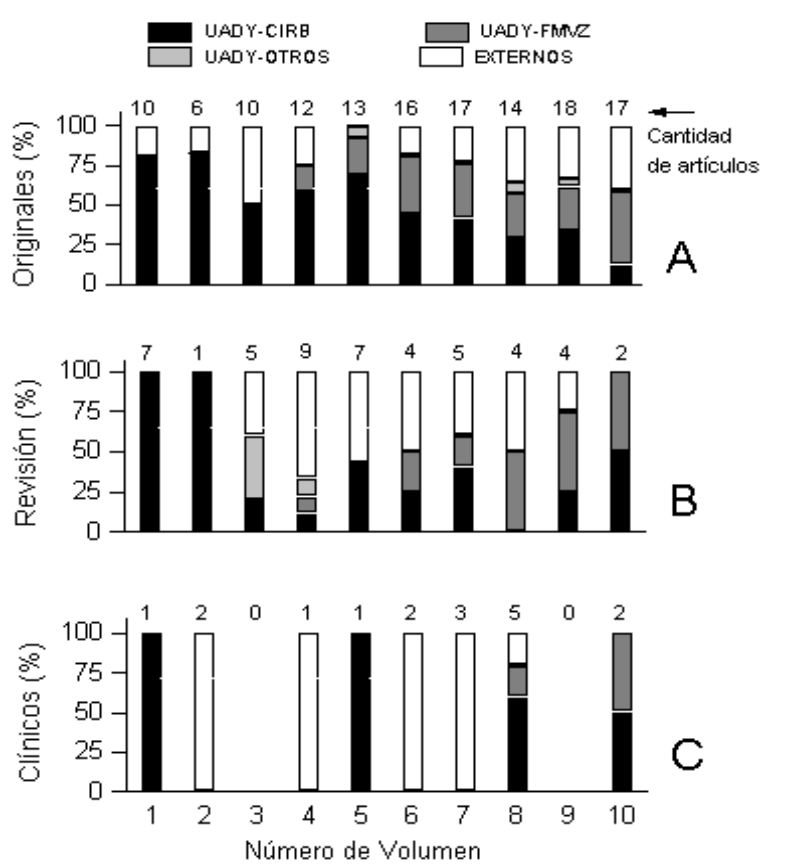

Figura 3.- Composición del contenido de los primeros diez volúmenes anuales de la Revista Biomédica. La parte A se refiere a los artículos originales, la B a los artículos de revisión y la $\mathrm{C}$ a los artículos de temas clínicos. En los tres casos se muestra el porcentaje de artículos para cada volumen escritos por autores de las dependencias señaladas arriba de las gráficas. También en la parte superior de las columnas se indica la cantidad de artículos de cada categoría publicados en cada volumen.

respuestas), edición y presentación (10 respuestas), revisores y Comité Editorial (8 respuestas), y difusión (6 respuestas). Cabe señalar que algunos encuestados opinaron sobre más de uno de estos aspectos. Por otro lado, hubo algunos suscriptores que sólo respondieron con un mensaje de cortesía o con comentarios demasiado generales y no fueron incluidos en el desglose anterior.

\section{Análisis de los artículos publicados.}

La figura 3 muestra el concentrado de resultados sobre los artículos publicados de acuerdo con la adscripción del autor principal. Puede verse que en sus inicios, más del 75\% de los artículos originales (gráfica A) publicados en la Revista Biomédica era de autores adscritos al CIR y que este nú- mero se fue reduciendo paulatinamente, hasta que en el volumen 10 la cifra se redujo a menos del $25 \%$. A partir del volumen 4 comenzaron a aparecer contribuciones de autores de la Facultad de Medicina Veterinaria y Zootecnia de la UADY, incrementándose gradualmente hasta representar poco más del $40 \%$ en el volumen 10 . Las contribuciones de autores externos a la UADY han sido variables, pero se han mantenido entre el 30 y $40 \%$ en los volúmenes 8,9 y 10 . En cuanto a la procedencia de los autores de los artículos de revisión (gráfica B), que fue la segunda categoría en número de artículos publicados, tuvieron una evolución similar a los artículos originales, aunque con más fluctuaciones. Los artículos sobre aspectos clínicos (gráfica C) fueron menos numerosos, con ninguna publicación de esta categoría en dos volúmenes y un máximo de 5 artículos publicados en el volumen 8. La procedencia de los autores de estos artículos ha sido la más variable de todas.

Análisis de la composición del Comité Editorial.

En la Figura 4 se presenta la composición (número de integrantes y sus lugares de adscripción) y evolución (por volúmenes anuales) del Comité Editorial. Puede verse que en el transcurso de sus primeros 10 años, el número de integrantes del Comité Editorial se incrementó de 24 a 35. Cabe destacar que este incremento se debió en su mayor parte a la incorporación de miembros procedentes de instituciones ajenas a la UADY, que pasaron de 8 (33\%) en el volumen 1, a 18 (51\%)en los volúmenes 9 y 10 . Asimismo, el número total de instituciones representadas en el Comité pasó de 5 en el volumen inicial a 17 en los dos volúmenes más recientes.

\section{Análisis de la participación interna de árbitros.}

En el número 4 del volumen 4 de la Revista Biomédica aparece por primera vez una nota de agradecimiento a los revisores. De ahí se desprende que de un total de 30 revisores que participaron en la calificación de artículos para los primeros cuatro volúmenes de la revista, 23 (77 \%) estaban afiliados al CIR o a la Facultad de Medicina. En el

\section{Revista Biomédica}


Análisis de la Revista Biomédica.

Vol. 5, de los 31 árbitros, 18 (58\%) eran de estas dependencias. Para el volumen 6, 21 (68\%) de 31 revisores eran internos, y en el Vol. 7, 18 (82\%) de 22. A partir del volumen 8 dejó de publicarse la nota de agradecimiento a revisores.

\section{Obtención de datos complementarios.}

De los trabajos publicados en la Revista Biomédica, Pudieron ubicarse las siguientes 15 citas en diversas revistas:

3 en Revista Do Instituto de Medicina Tropical de Sao Paulo (Brasil)

2 en Revista de Investigación Clínica (México) 2 en Memorias Do Instituto Oswaldo Cruz (Río de Janeiro, Brasil)

1 en Ciencia y Desarrollo (México)

1 en Ginecología y Obstetricia de México

1 en Veterinaria México

1 en Journal of Medical Entomology

1 en Agrociencia

1 en Preventive Veterinary Medicine 1 en Archivos de Neurociencias (México)

1 en Veterinary Parasitology

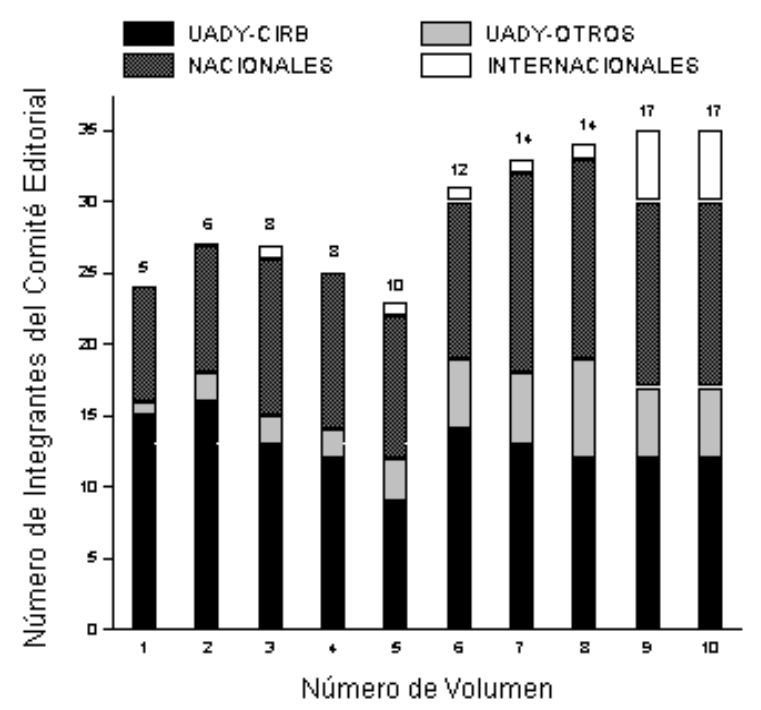

Figura 4.- Composición y evolución del Comité Editorial de la Revista Biomédica. Para cada volumen anual se muestra el número de integrantes según las cuatro categorías de adscripción indicadas. En la parte superior de cada columna aparece el número de instituciones representadas en el Comité para ese volumen.
Cabe aclarar que alrededor de la tercera parte de estas referencias son autocitas. Asimismo, se localizaron dos referencias en libros especializados sobre artículos aparecidos en la Revista Biomédica. En algunos casos, los encuestados respondieron que sí habían hecho referencia en otras publicaciones a sus artículos de la Revista Biomédica, pero lamentablemente no aportaron los datos. También cabe señalar que algunos autores citaron sus propios trabajos publicados en la Revista Biomédica en números posteriores de la misma revista. Estos últimos no se incluyen en la tabla anterior. Por otra parte, algunos encuestados que han sido autores de artículos en la Revista Biomédica, mencionaron que han hecho referencia a sus artículos en revistas regionales, tales como la Revista Yucateca de Pediatría.

Porcentajes de aceptación y rechazo de los artículos sometidos a evaluación.

El editor de la Revista Biomédica menciona que fue aceptado el $82 \%$ de todos los artículos sometidos a la revista en el periodo de enero de 1990 a junio de 1999 (7). La única razón especificada para el rechazo es que, según los árbitros, los artículos no estaban de acuerdo con la política editorial de la revista. En la misma fuente, el Dr. Norberto Sotelo Cruz, editor del Boletín Clínico del Hospital Infantil del Estado de Sonora, indica que en esa publicación se rechaza entre el 30 y el 35\% de los artículos recibidos. Por su parte, el Químico Alvar Loría Acereto, de la Revista de Investigación Clínica, asevera que esa publicación rechaza el 32\% de las contribuciones que recibe.

\section{DISCUSIÓN.}

\section{Encuesta.}

Con respecto a los resultados derivados de la encuesta, se pueden hacer algunas observaciones. Un primer hallazgo, hasta cierto punto predecible, es que de los lectores que respondieron el cuestionario, poco más de la tercera parte labora

Vol. 11/No. 4/Octubre-Diciembre, 2000 


\section{FJ Álvarez-Cervera, JL Góngora-Alfaro, JL Bata-García, FJ Heredia-López.}

en el Centro de Investigaciones Regionales de la UADY, donde se edita la Revista Biomédica (figura 1A). De hecho, un porcentaje elevado $(76 \%)$ de este grupo de encuestados ha sido autor de uno o varios artículos publicados en la Revista Biomédica. Otro tercio de los encuestados trabaja en instituciones de la República Mexicana situadas fuera de Mérida.

Como segundo punto está el hecho de que cerca de la mitad de los lectores que respondieron señalaron como una de sus actividades profesionales la de la investigación (figura 1B). Y casi otro $25 \%$ correspondió a personal clínico. Es decir, el grueso de los lectores de la Revista Biomédica se clasifica en una de estas dos categorías o en ambas.

Casi la mitad de los suscriptores indicaron que lee de uno a tres artículos de cada número (figura 1C). De estos, 51 lectores son de la UADY y 41 son externos. Además, de 27 lectores que respondieron que leen la mayoría de los artículos, 17 son ajenos a la UADY. Si esto pudiese extrapolarse a toda la población de suscriptores, entonces este dato sugiere que el contenido de los artículos despierta el interés de un número importante de lectores a los que se envía la revista.

En cuanto a la preferencia de los lectores por los tipos de artículos, se encontró que poco más de la cuarta parte lee los escritos originales de la Revista Biomédica, y casi una quinta parte acostumbra leer los artículos de revisión (figura 1D). Los casos clínicos fueron los artículos que siguieron en orden de preferencia. Estos datos podrían insinuar que estos tipos de artículos son los que deberían predominar marcadamente en el contenido de la revista.

Alrededor de dos terceras partes de los lectores comparten sus ejemplares con otras personas (figura 1E). Este hecho sugeriría que existe un número importante de suscriptores potenciales.

Otro detalle positivo es que alrededor del $40 \%$ de los lectores conserva todos los números de la revista (figura 1F). Esto indicaría que el contenido de la publicación resulta de utilidad para muchos lectores.

Un valor cercano al $40 \%$ de los lectores ha sido autor de al menos una contribución a la Revista Biomédica (figura 2A). De estos, 50 están afiliados a la UADY y 7 no. Esta cifra podría interpretarse como un reflejo del papel que juega la revista como medio de intercambio de información, particularmente en el medio institucional, y en menor grado, a nivel regional y nacional. Por otra parte, podría inferirse que los autores de la revista predominan entre quienes respondieron al cuestionario simplemente porque desean involucrarse más en el progreso de la Revista Biomédica.

En contraparte, prácticamente la mitad de los encuestados nunca ha sido autor en la propia revista, aunque no descartaría serlo. Esta cifra representaría, además, al grupo de usuarios o beneficiarios de la revista. Es decir, quienes recurren a ella para mantenerse al día, para hacer una consulta o por otros intereses académicos o profesionales. En ese sentido, sería deseable incrementar este porcentaje a fin de extender la cobertura de la revista. Finalmente, son muy pocos los lectores (4.9\%) que nunca han publicado en la revista ni piensan hacerlo en el futuro. Esto podría deberse a que algunos suscriptores no solicitaron recibir la revista por no tener una afinidad específica con los temas que se abordan en ella, o bien, porque sus propias actividades e intereses han cambiado con el paso del tiempo.

A pesar de que la Revista Biomédica no aparece actualmente en bases de datos bibliográficas mundiales del área médica, se pudieron obtener algunos datos de las citas aparecidas en otras publicaciones, algunas de primer nivel, así como en libros. Además, el cuestionario incluyó una pregunta a este respecto. Prácticamente el 15\% de los encuestados afirmó que ha citado sus trabajos en otras revistas (y proporcionó las citas correspondientes). Dado que no todas las referencias estaban completas, sólo podemos estimar que alrededor de la mitad de estas referencias eran autocitas. Por otro lado, más de la mitad de los

\section{Revista Biomédica}


Análisis de la Revista Biomédica.

encuestados nunca ha citado sus propios trabajos (figura 2B). Podría suponerse que esto obedece al hecho de que los trabajos publicados en la Revista Biomédica, por su frecuente carácter regional, no tuvieran relación directa con los estudios reportados en otras publicaciones, presumiblemente de mayor cobertura.

Una pregunta crucial es la referente a la opinión de los lectores respecto a la calidad de la revista en términos generales. $\mathrm{Y}$ el resultado encontrado fue que casi las dos terceras partes (el $62 \%$ ) estima que la revista es buena, mientras que el $19 \%$ piensa que es excelente y el $11 \%$ la considera regular (figura $2 \mathrm{C}$ ). Ningún lector opinó que la calidad de la Revista Biomédica fuera mala.

El asunto de publicar la revista únicamente en formato electrónico tuvo una reacción contundente: más del $70 \%$ de los lectores dictaminó en contra de esta idea. Sólo el $16.9 \%$ votó a favor de una publicación hecha exclusivamente en medios electrónicos, y un $8.4 \%$ se expresó como indeciso en esta cuestión (figura 2D). De todas formas, al igual que muchas otras revistas científicas, la Revista Biomédica también se publica en formato electrónico in extenso a partir del volumen 8 (1997). Los lectores interesados pueden consultarla en la siguiente dirección: http://www.uady.mx/sitios/biomedic/ revibiom.html.

Las respuestas con respecto a la posibilidad de pagar una cuota por recibir la revista indican que la gran mayoría (más de las tres cuartas partes) de los suscriptores (en este caso, tanto lectores personales como bibliotecas) estarían dispuestos a cubrir una cuota (figura 2E). Este es un indicador del valor que los lectores asignan al material que aparece en la Revista Biomédica. De las siete bibliotecas que respondieron la encuesta, sólo una se interesó por la opción de corresponder a la suscripción a la Revista Biomédica con un canje de publicaciones. Es interesante señalar que el responsable de esta biblioteca reportó que la Revista Biomédica había sido consultada en sala un total de 1496 veces en el período de 1996 a
1998 (3 años). Por otro lado, a partir del número 2 del volumen 11 de la Revista Biomédica se incluye un cupón de suscripción anual (4 números) en el que se especifica un costo de $\$ 250$ pesos mexicanos o $\$ 40$ dólares americanos.

Finalmente, al no haberse acotado los temas para los comentarios solicitados en los cuestionarios, hubo una gran diversidad de opiniones, a veces encontradas. Sin embargo, se pueden extraer los siguientes puntos relevantes a cada uno de los cuatro temas señalados anteriormente (contenido de la revista, edición y presentación, revisores y Comité Editorial, y difusión).

Con respecto a la temática hubo quien planteó que se redujera el espectro de materias comprendidas en el perfil de interés de la revista. Igualmente, hubo lectores que recomendaron lo contrario. También se sugirió que se incrementara el número de artículos originales, así como los de revisión. Esto concuerda con las preferencias de los lectores con respecto a los tipos de artículos que más leen los suscriptores.

Asimismo, varios lectores opinaron que la revista se debería orientar exclusivamente a asuntos relacionados con medicina humana, mientras otros consideraron que debía de cubrir todas las áreas biológicas. Una sugerencia que podemos hacer al respecto es que se dedique un número al año a un campo en particular, por ejemplo, enfermedades infecciosas. Para este fin, y con suficiente anticipación, se haría el anuncio de esta intención y se solicitarían artículos acordes con el área. Otro número podría comprender únicamente artículos sobre temas de veterinaria y zootecnia, otro sobre neurociencias, y así sucesivamente. Una idea más, manifestada por un encuestado es la de crear periódicamente suplementos dedicados a temas no relacionados con el ser humano.

Con referencia a la publicación en sí, surgieron las siguientes propuestas: que los artículos sean publicados exclusivamente en inglés, que se inserte publicidad pagada en la revista (relacionada con medicina), que se mejore la revisión lingüística y tipográfica, que se recabe la 


\section{FJ Álvarez-Cervera, JL Góngora-Alfaro, JL Bata-García, FJ Heredia-López.}

opinión de los lectores con mayor frecuencia y más ampliamente, que se añadan ilustraciones a color, etc. Este último punto ya ha sido incluido desde el número 3 del volumen 10 (julio-septiembre de 1999).

En cuanto a los miembros del Comité Editorial y los revisores, se sugirió que éstos sean investigadores activos, con el fin de mejorar los arbitrajes y seleccionar más rigurosamente los artículos publicables. También se propuso que en el Comité Editorial se incluyera a miembros que, por su área de especialización, cubrieran todo el espectro de temas de interés para la revista.

Acerca de la difusión de la revista, cabe señalar que hay dos preocupaciones: incrementar el número de lectores y atraer contribuciones de otros lugares y de mayor calidad. Para lograr esto, un plan sugerido es el de promocionar la publicación entre las asociaciones médicas, desde las locales hasta las internacionales. Otra idea es la de eliminar las suscripciones gratuitas a lectores individuales $\mathrm{y}$, en cambio, promover las suscripciones sin cargo para bibliotecas.

Análisis de los artículos publicados.

De la figura 3 se desprende que, en un inicio, la gran mayoría de los artículos originales y de revisión correspondían, como era posible suponer, a autores del CIR. Más adelante, aumentaron notoriamente las contribuciones externas, en especial en lo referente a artículos de revisión. A partir del cuarto volumen, la Facultad de Medicina Veterinaria y Zootecnia (FMVZ) de la UADY inició sus aportaciones a la revista, tanto de artículos originales como de revisión, las cuales gradualmente se fueron incrementando hasta convertirse en mayoría en los volúmenes más recientes. Este incremento se vio acompañado de una disminución en las contribuciones de académicos del CIR. La situación final (volumen 10) refleja que el $47 \%$ de los artículos originales fueron obra de autores de la FMVZ, mientras que sólo el $12 \%$ estuvieron firmados por colaboradores del CIR. En ese mismo volumen sólo se incluyeron dos artículos de revisión: uno por parte de la
FMVZ y otro del CIR. Este cambio de contenido, predominantemente sobre temas médicos en el comienzo, y luego con mayor abundancia en escritos relacionados con veterinaria, ha sido un motivo de la polémica que se ha manifestado en los comentarios de los lectores encuestados.

Con excepción del CIR y la FMVZ, las contribuciones de artículos de autores adscritos a otras dependencias de la UADY han sido muy escasas. De hecho, los artículos originales sólo estuvieron presentes en tres volúmenes, llegando a representar como máximo el 8\% (1 artículo) del contenido de un volumen. Algo similar ocurrió con los artículos de revisión, pues en ocho de los diez volúmenes considerados, no hubo ninguna colaboración de autores adscritos a otras dependencias de la UADY, con la excepción del volumen 3 en el que dos (40\%) de las cinco revisiones fueron de autores de la Facultad de Medicina. Estas cifras son muy elocuentes e indican que es necesario fomentar las aportaciones de académicos de las Facultades de Medicina, Odontología, Enfermería y Química de la UADY.

En el caso de autores de instituciones externas a la UADY, su contribución en el contenido de la Revista Biomédica ha sido muy variable, pero ha sido de mayor peso en artículos de revisión. Los autores externos contribuyeron con artículos de revisión en siete volúmenes, alcanzando un máximo (66.6\%) en el volumen 4 (seis revisiones de un total de nueve). En cuanto a los artículos originales, los autores externos han contribuido en nueve volúmenes, con un máximo (50\%) en el volumen 3 (cinco de un total de diez). Como puede apreciarse en la figura 3 , el número de artículos originales externos ha ido en aumento a partir del volumen 6 , mientras que la cantidad de artículos de revisión ha tendido a bajar en los últimos años. Aunque estas cifras son alentadoras, sería deseable implementar mecanismos para aumentar el número de contribuciones originales de autores externos a la UADY.

Una información adicional es la que GóngoraBiachi menciona en el sentido de que en 1999 alrededor del 55\% de los artículos publicados eran

\section{Revista Biomédica}




\section{Análisis de la Revista Biomédica.}

de Yucatán, 30\% de otras partes del país y 15\% de Centro y Sudamérica (7).

\section{Análisis de la composición del Comité Editorial.}

En cuanto a la composición del Comité Editorial, puede observarse en la figura 4 que tanto el número de sus miembros como la variedad de instituciones que representan se ha ido incrementando a través del tiempo. Esto es importante y debe continuar esta política de diversificación. Por otra parte, es entendible que inicialmente en la composición del Comité hayan predominado los revisores de las instituciones sede de la revista. Pero debe también cuidarse el aspecto de contar con un Comité caracterizado por una representación balanceada de acuerdo con los temas que se manejen en la revista. Asimismo, debe contemplarse algún mecanismo para la selección adecuada de sus miembros, así como la aplicación de lineamientos que aseguren su participación y nivel de competencia o su sustitución. Idealmente, debe reclutarse para la labor editorial a los investigadores más destacados en cada campo de las ciencias biomédicas. Esto último puede establecerse con base en el número de publicaciones internacionales con arbitraje de cada uno de ellos. Sin embargo, el contar con un comité editorial integrado por científicos destacados, como señala Pannell (8), no garantiza un contenido con una calidad a la par. En concordancia con esta observación, este autor sugiere requerir a los miembros del comité que publiquen en la propia revista de vez en cuando. Después de todo, junto con el editor, este cuerpo es responsable de la calidad editorial de la revista y de tareas específicas como la selección de árbitros.

\section{Análisis de la participación interna de árbitros.}

En los primeros siete volúmenes de la revista, fue preponderante la actuación de árbitros del CIR y de la Facultad de Medicina (entre el 58 y el $82 \%$ de total, como se indicó). Afortunadamente, esta tendencia se ha invertido, pues más recientemente alrededor del 60\% de los árbitros son de fuera del estado de Yucatán, y de éstos, el 20\% son del extranjero (7). Es claro que con frecuencia será necesario recurrir a especialistas foráneos para calificar artículos de una creciente gama de temas biomédicos. Podría incorporarse en las Instrucciones para los Autores, la indicación de que es aceptable que los propios autores sugieran árbitros para sus contribuciones. Asimismo, los miembros del Comité Editorial deberían auxiliar al Editor, haciendo recomendaciones sobre posibles árbitros que estén calificados en disciplinas especializadas.

\section{Referencias a artículos publicados en la Revista} Biomédica

Un comentario prometedor acerca de las referencias hechas a artículos publicados en la Revista Biomédica es la inminente inclusión de la revista en índices como SCIELO (Scientific Electronic Library Online). Este índice, a su vez, será incorporado a la base de datos Medline y esto permitiría una mayor difusión de los artículos que aparecen en la Revista Biomédica y la posibilidad de hacerse de un factor de impacto propio y realista, algo que interesa a los autores.

\section{Datos complementarios.}

Por último, un factor que será determinante en el progreso de la revista será el de depurar su contenido en función de una selección más rigurosa de los escritos que recibe para consideración editorial. El caso de las tasas de aceptación de trabajos de la prestigiosa revista Science es ilustrativo: tratándose de escritos provenientes de Estados Unidos, en 1994 era del 21\%, mientras que para manuscritos provenientes de países en desarrollo era de sólo 1.4\% (3). ¿Quiere decir esto que existe una discriminación o hay razones fundamentadas que lo respalden? Las opiniones pueden ser opuestas entre sí, pero lo que podría servir de guía para mejorar la calidad de una revista es tomar en cuenta los puntos que se suelen criticar a los trabajos de naciones del tercer mundo, y que pueden corregirse. Se dice que la investigación reportada desde estos países es con frecuencia anticuada, mal hecha, con una documentación pobre, con méto- 


\section{FJ Álvarez-Cervera, JL Góngora-Alfaro, JL Bata-García, FJ Heredia-López.}

dos que no cumplen con las normas aceptadas y con errores idiomáticos de todo tipo (ortografía, sintaxis, semántica, puntuación) (3).

De cualquier manera, se antoja que un $18 \%$ de artículos rechazados por la Revista Biomédica es una cifra demasiado baja, sobre todo cuando se le compara con los porcentajes de otras revistas biomédicas mexicanas, que son de alrededor del $32 \%$.

\section{Otras Observaciones y Recomendaciones.}

Una primera sugerencia es la de eliminar las suscripciones gratuitas personales, cuando menos en el caso de los académicos de la UADY. Sentimos que con ofrecer una suscripción por departamento sería suficiente para alcanzar una adecuada cobertura de lectores internos. Los ejemplares que así quedaran disponibles podrían ofrecerse a bibliotecas públicas a través de suscripciones gratuitas.

Algunas formas en las que se podrían aplicar los ingresos generados por concepto de suscripciones pagadas serían: promover suscripciones a costo reducido para estudiantes, incrementar el número de páginas de la revista, y/o aumentar la cantidad de ilustraciones a color.

Otra sugerencia sería la de conseguir la inclusión de la Revista Biomédica en el Índice de Revistas Mexicanas de Investigación Científica y Tecnológica del Consejo Nacional de Ciencia y Tecnología (CONACyT) (9). Esto contribuiría a mejorar la "visibilidad" de la revista en el extranjero, se facilitaría la obtención de apoyo económico por parte del propio CONACyT, y serviría como una referencia más de su calidad y de su impacto. Además, algunas instituciones académicas ya lo toman en cuenta para evaluar los productos de sus investigadores. Por otra parte, el criterio más importante que se utiliza para determinar el ingreso al índice es la calidad del proceso de arbitraje. En consecuencia, la evaluación por pares en la Revista Biomédica debería revisarse y adecuarse previamente, de tal manera que los árbitros fueran siempre especialistas reconocidos (nacionales y extranjeros) y que rutinariamente calificaran los trabajos en forma documentada, asegurando así la originalidad de los mismos. Con esta evaluación más rigurosa, se esperaría un incremento en el factor de impacto de los trabajos. Y, a final de cuentas, es este parámetro el elemento más utilizado para aquilatar la calidad de las revistas científicas. Se considera que las revistas incluidas en el índice de CONACyT (62 de ellas en 1999) podrían llegar a ser incorporadas al SCI. Y una ventaja más de este índice es que agrupa a las revistas por áreas, eliminándose así algunas generalizaciones improcedentes. Los otros requisitos comunes para ingresar a índices de prestigio, la periodicidad y la distribución, han sido cubiertos ya por la Revista Biomédica. Es importante destacar que todos los números han sido publicados trimestralmente y de manera ininterrumpida durante más de 10 años.

En suma, consideramos que el esfuerzo invertido en la Revista Biomédica es encomiable y debe mantenerse. Su éxito dependerá, en buena medida, de su capacidad por atraer a una mayor cantidad de autores, teniendo así la posibilidad de elegir para publicación sólo las contribuciones más sobresalientes y de mayor relevancia. Esto, a su vez, hará que crezca el número de lectores y de las citas a los trabajos que aparezcan en ella.

\section{AGRADECIMIENTOS.}

Expresamos nuestro agradecimiento al Dr. Renán Góngora Biachi y al Br. Luis Escobedo y Ruiz por darnos acceso a la base de datos de los suscriptores de la Revista Biomédica y por el apoyo que nos brindaron de múltiples maneras en la realización de la encuesta y en otros aspectos del presente trabajo. Asimismo, la ayuda brindada por el Dr. Fernando Puerto Manzano, director del CIR, resultó invaluable para la obtención de la información de los lectores. Finalmente, agradecemos a todos los lectores y bibliotecarios que respondieron a la encuesta, parte fundamental de nuestro estudio.

\section{REFERENCIAS.}

1. Zavala-Velázquez, JE. Editorial. Rev Biomed 1990; 1:3.

2. Góngora-Biachi, RA. Análisis de la Revista Biomédica (Editorial). Rev Biomed 1995; 6:1-4.

\section{Revista Biomédica}


3. Wayt Gibbs, W. Lost science in the third world. Scientific American 1995; 273:76-83.

4. Martínez-Chacón, ML. Publicaciones Científicas en España. Reflexiones de una editora en el campo de las ciencias de la naturaleza. Carta al editor. Rev Biomed 1996; 7:247-248.

5. Góngora-Biachi, RA. La Revista Biomédica: una década de publicación. Rev Biomed 1999; 10:197-198.

6. Artemisa 4. Artículos editados en México sobre información en salud. Información científica nacional sobre salud en CD-ROM. El contenido completo de las 28 mejores revistas biomédicas publicadas en México durante 1995. Centro Nacional de Información y Documentación Sobre Salud. Secretaría de Salud. México. 1996

7. Góngora-Biachi, RA. Experiencia en la edición de la Revista Biomédica. Boletín de la AMERBAC 1999; 2:4-5.

8. Pannell, K. La ética en las ciencias: el caso de las revistas científicas. Avance y Perspectiva 2000; 19:179-184.

9. Pérez Angón, M. A. Revisión de dos libros "Revistas científicas en América Latina", Cetto, A.M. y Alonso, O. (compl.), FCE, 1999, y "Los dilemas de las revistas académicas mexicanas", Loría Díaz E. (compl.), UAEM, 1999. Avance y Perspectiva 2000; 19:253-258. 